\title{
Finite Correlation Length Scaling with Infinite Projected Entangled-Pair States
}

\author{
Philippe Corboz, ${ }^{1}$ Piotr Czarnik, ${ }^{2}$ Geert Kapteijns, ${ }^{1}$ and Luca Tagliacozzo ${ }^{3}$ \\ ${ }^{1}$ Institute for Theoretical Physics and Delta Institute for Theoretical Physics, University of Amsterdam, \\ Science Park 904, 1098 XH Amsterdam, Netherlands \\ ${ }^{2}$ Institute of Nuclear Physics, Polish Academy of Sciences, \\ Radzikowskiego 152, PL-31342 Kraków, Poland \\ ${ }^{3}$ Department of Physics and SUPA, University of Strathclyde, Glasgow G4 ONG, United Kingdom \\ and Departament de Física Quàntica i Astrofísica, Universitat de Barcelona, Barcelona 08028, Spain
}

(Received 28 March 2018; revised manuscript received 12 June 2018; published 30 July 2018)

\begin{abstract}
We show how to accurately study two-dimensional quantum critical phenomena using infinite projected entangled-pair states (iPEPS). We identify the presence of a finite correlation length in the optimal iPEPS approximation to Lorentz-invariant critical states which we use to perform a finite correlation length scaling analysis to determine critical exponents. This is analogous to the one-dimensional finite entanglement scaling with infinite matrix product states. We provide arguments why this approach is also valid in $2 \mathrm{D}$ by identifying a class of states that, despite obeying the area law of entanglement, seems hard to describe with iPEPS. We apply these ideas to interacting spinless fermions on a honeycomb lattice and obtain critical exponents which are in agreement with quantum Monte Carlo results. Furthermore, we introduce a new scheme to locate the critical point without the need of computing higher-order moments of the order parameter. Finally, we also show how to obtain an improved estimate of the order parameter in gapless systems, with the 2D Heisenberg model as an example.
\end{abstract}

DOI: 10.1103/PhysRevX.8.031031

\section{INTRODUCTION}

In recent years there has been a very active development of tensor network variational ansätze for describing strongly correlated quantum many-body systems [1-5]. Tensor networks exploit the fact that ground states and lowenergy states of local Hamiltonians are typically only weakly entangled, where the entanglement entropy of a region scales only with its surface rather than with its volume- a property known as the area law of entanglement [6-11]. As a consequence, the information contained in these states can be compressed and described by using specific tensor networks where the elementary tensors have finite bond dimension $D$, such as one-dimensional matrix product states (MPS) [12-14] and 2D projected entangledpair states (PEPS) $[1,15,16]$ (also called tensor product states $[17,18])$.

In particular, in 1D, infinite MPS (iMPS) have successfully been used to characterize the universal properties of critical systems. This at first sight seems counterintuitive

Published by the American Physical Society under the terms of the Creative Commons Attribution 4.0 International license. Further distribution of this work must maintain attribution to the author(s) and the published article's title, journal citation, and DOI.
Subject Areas: Computational Physics,

Quantum Information,

Strongly Correlated Materials since 1D critical states violate the area law of entanglement [19-23] while an iMPS with finite $D$ can only describe states fulfilling the area law, i.e., noncritical states with a finite correlation length $\xi[12-14]$. However, with increasing $D, \xi_{D}$ increases, improving the accuracy of the approximate state in a systematic way. As a result local observables follow the universal scaling laws characteristic of the underlying critical point. In the scaling regime $\xi_{D}$ acts as a cutoff on the exact, diverging correlation length, similarly to a finite system size. The possibility to tune $\xi_{D}$ by varying the bond dimension can be practically used to extract critical exponents in a very similar way as in standard finite-size scaling approaches. This powerful approach is known as finite entanglement scaling, and is also called finite correlation length scaling (FCLS) [24-27].

An important question is whether a similar approach can also be designed in 2D, which would be highly desirable since most critical exponents can only be computed numerically [28]. However, the situation in 2D seems different because unlike in 1D there exist critical states with an area law [35-41] and there are known examples of exact critical infinite projected entangled-pair states (iPEPS) with a finite $D[40,42]$. The latter include 2D classical states [43] and ground states of generalized Rokhsar-Kivelson (RK) Hamiltonians at their critical point. 
When the RK Hamiltonian is critical the low-energy excitations' energy-momentum dispersion relation is $E(k) \propto k^{z}$ with $z \geq 2$ and the RK states effectively describe the partition function of 2D classical models [41,44-51]. Besides RK states, it is currently still unclear whether a generic quantum critical 2D state, obeying the area law, can be exactly represented by finite- $D$ iPEPS.

Here we focus on a special case of quantum phase transition, a critical point with low-energy excitations exhibiting a linear energy-momentum dispersion relation, $E(k) \propto k$. The linear dispersion is the footprint of an enhanced emerging symmetry, where energy and momentum (or space and time) play a very similar role and thus these critical points are called Lorentz-invariant critical points. For such a critical point there are no known examples of a finite- $D$ iPEPS that exactly represents the critical state [51-53].

In this paper we provide arguments justifying that an iPEPS with finite $D$ cannot represent a Lorentz-invariant critical state exactly. The finite $D$ always induces a finite correlation length $\xi_{D}$ in the iPEPS state, in complete analogy to the 1D case. Lorentz-invariant critical points could thus describe a class of states which, despite fulfilling the area law of entanglement, cannot be faithfully represented by an iPEPS with a finite $D$ (see also Ref. [54]). As a positive consequence, this allows us to the apply the ideas of FCLS also in 2D for the accurate and systematic study of quantum critical phenomena.

In order to demonstrate the applicability and power of FCLS in 2D, we present state-of-the-art simulation results for interacting spinless fermions on the honeycomb lattice where we find critical exponents in agreement with quantum Monte Carlo (QMC) results. Furthermore, we introduce a new scheme to locate the critical point, based on the order parameter $m$ and its derivative (called the $\mathrm{m}^{\prime} / \mathrm{m}$ approach). Similar to the usual Binder cumulant approach, this scheme does not require the a priori knowledge of the critical exponents, but it is simpler since it is not based on higher moments of the order parameter, which are computationally expensive to obtain with iPEPS. We also show how FCLS can be used to obtained improved estimates of order parameters in gapless systems, with the 2D Heisenberg model as an example.

Our reasoning seems to indicate that the mismatch between finitely correlated iPEPS and critical states has a geometric origin. The finite $D$ in iPEPS, in the scaling regime, transforms the continuous space-time into a landscape of towers in imaginary time separated by valleys. The intertower separations are at the scale of the lattice spacing, but the finite correlation along the towers provides the infrared cutoff that is ultimately responsible for the appearance of the finite correlation length in the system, as sketched in Fig. 1.

This paper is organized as follows. Section II provides a brief introduction to iPEPS, followed by a heuristic

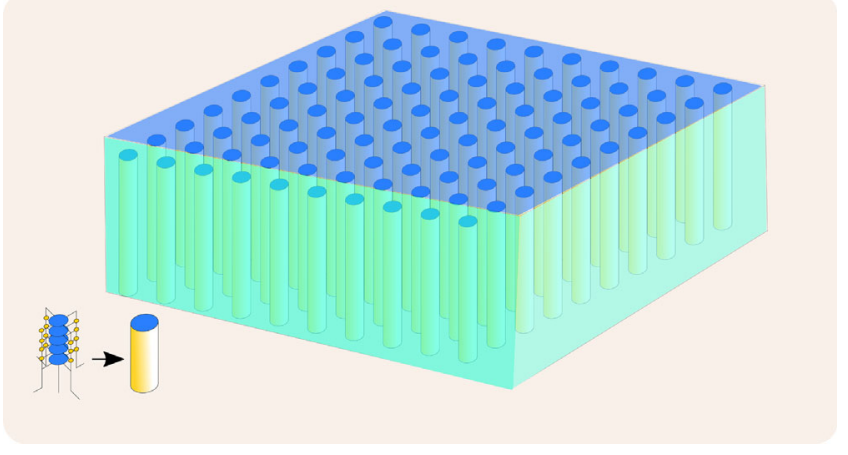

FIG. 1. In the scaling regime, close to a Lorentz-invariant critical point, the low-energy physics of a $2 \mathrm{D}$ quantum lattice system is described by a continuous $3 \mathrm{D}$ field theory. In the same regime, the iPEPS wave function describes a more complicated landscape, where the 3D space is pierced by infinitely long valleys separated by distances of the order of the lattice spacing. The correlation length along those towers provides an infrared cutoff, as if the system had a finite imaginary correlation time, leading to a finite spatial correlation length, which we observe in our numerical simulations. These towers are generated by the finite bond dimension in iPEPS (shown in the lower left-hand corner of the illustration), as explained in detail in the main text.

discussion about the effects of encoding the ground state of a 2D Hamiltonian at a Lorentz-invariant quantum critical point with an iPEPS in Sec. III. In Sec. IV, we explain how to perform a FCLS analysis with iPEPS in practice. In Sec. V, we present our numerical results for the interacting spinless fermion model and explain the $\mathrm{m}^{\prime} / \mathrm{m}$ scaling approach. The extrapolation technique based on FCLS to improve the extrapolation for the order parameter to its exact infinite- $D$ value is explained in Sec. VI. Finally, in Sec. VII, we summarize our main findings and conclusions.

\section{INFINITE PROJECTED ENTANGLED-PAIR STATES}

An iPEPS $[1,15,16]$ (also called a tensor product state $[17,18])$ is an efficient variational tensor network ansatz for two-dimensional ground states of local Hamiltonians in the thermodynamic limit, and can be seen as a natural generalization of (infinite) matrix product states to two dimensions. The ansatz consists of a periodically repeated unit cell of tensors with one tensor per lattice site. In the present work, we used a cell with 2 tensors arranged in a checkerboard pattern. Each tensor has one physical index, carrying the local Hilbert space of a lattice site, and 4 (3) auxiliary indices on a square (honeycomb) lattice. As in a MPS, the accuracy of the ansatz is systematically controlled by the bond dimension $D$ of the auxiliary indices. In recent years iPEPS has become a very powerful approach which has been applied to a broad range of challenging problems, including frustrated spin systems and strongly correlated electron systems; see, e.g., Refs. [41,55-66] and references therein. 
The optimization of the tensors (i.e., finding the optimal variational parameters) in this work is done by first performing an imaginary time evolution [67-69] and then further improving the state using a variational optimization [70] (see also Ref. [71]). The contraction of the 2D tensor network is done using a variant [60] of the corner transfer matrix (CTM) method [72,73], where the accuracy of the contraction is controlled by dimension $\chi$ of the boundary tensors; see, e.g., Ref. [67] for details. In order to improve the efficiency we exploited global Abelian symmetries in the tensor network ansatz [74,75].

\section{LORENTZ-INVARIANT CRITICAL POINTS}

Lorentz-invariant critical points are points in the phase diagram of a many-body systems in which (i) the Hamiltonian is gapless and (ii) the low-energy excitations' dispersion relation depends linearly on the momentum $k$, $E(k)=v k$, where $E$ is the energy of the excitation and $v$ is the sound velocity.

The linear dispersion relation implies that the system at low energy becomes Lorentz invariant. The Lorentz transformations mix space and time. In order to understand the role of "time" in the ground state of a critical system it is better to appeal to the universality of the critical point. In this way we can describe the same scenario in terms of a classical 3D system where the extra dimension will represent the time. The low-energy emerging properties at the critical point of both the 2D quantum system and the 3D classical system are the same, provided that we choose a critical 3D classical system in the same universality class as the 2D quantum system.

One way to construct a 3D classical system in the same universality class of our 2D quantum system is through the correspondence between classical and quantum mechanics. From the partition function of a classical model we can write the ground-state space projector $|\Omega\rangle\langle\Omega|=$ $\lim _{\beta \rightarrow \infty} \exp (-\beta H) / Z$, with $Z=\operatorname{tr}[\exp (-\beta H)]$ [76], and the ground-state expectation values $\langle\mathcal{O}\rangle=\operatorname{tr}(|\Omega\rangle\langle\Omega| \mathcal{O})$, with $\mathcal{O}$ an arbitrary operator. This is done by (i) dividing the Euclidean time $\beta$ into many small intervals, $\beta / N_{t}=\delta \beta$. In this way (ii) the expectation value of an operator is written in terms of a large power of a certain operator $\langle\mathcal{O}\rangle=\operatorname{tr}\left(T^{N_{t}} \mathcal{O}\right)$, where $T=\exp (-\delta \beta H)$. We (iii) insert the resolution of the identity in a preferred basis of the Hilbert space $\mathbb{I}=\sum_{\{n\}}|\{n\}\rangle\langle\{n\}|$ before and after each $T$. This allows us to identify a "classical" transfer matrix. (iv) As a consequence of the locality of the Hamiltonian $H$ and the fact that $\delta \beta \ll 1, T$ can be expressed approximately (with an error that scales to zero as $\delta \beta \rightarrow 0$ faster than $\delta \beta^{2}$ ) as the contraction of local Boltzmann weights, by, for example, performing a Trotter-Suzuki expansion of $T$ together with a singular value decomposition of the resulting local terms [77-79]. As a result we can express the ground space projector of a 2D Hamiltonian (and consequently all ground-state expectation values) as the infinite contraction of a simple 3D tensor network.

The construction holds for any 2D quantum system and becomes exact in the limit $\delta \beta \rightarrow 0$. The emerging Lorentz symmetry close to a Lorentz-invariant critical point allows us to exchange the role of the Euclidean time $(\beta)$ with any of the space directions. As a consequence, the correlation length in the time direction is proportional to the correlation in the space direction $\xi_{s}=v \xi_{t}$, where $v$ is the velocity appearing in the low-energy dispersion relation and $\xi_{s}$ and $\xi_{t}$ denote the correlation length and correlation time, respectively.

We now give an argument in favor of the fact that by encoding the 3D tensor network in a 2D iPEPS with finite bond dimension we force the correlation time $\xi_{t}$ to be finite.

\section{A. Finite correlation time}

The easiest way to understand why a finite $D$ induces a finite correlation time is to represent a $2 \mathrm{D}$ cut of the $3 \mathrm{D}$ tensor network in which we represent only $x, t$ of the $x, y, t$ coordinates. We describe the process of encoding the $3 \mathrm{D}$ network in a 2D iPEPS as if we were actually performing an imaginary time evolution. The $2 \mathrm{D}$ cut of the infinite tensor network is represented in Fig. 2. There, as usual, tensors are represented by geometric shapes and the lines attached to them represent their indices. Lines connecting two tensors encode the contraction of the two tensors with

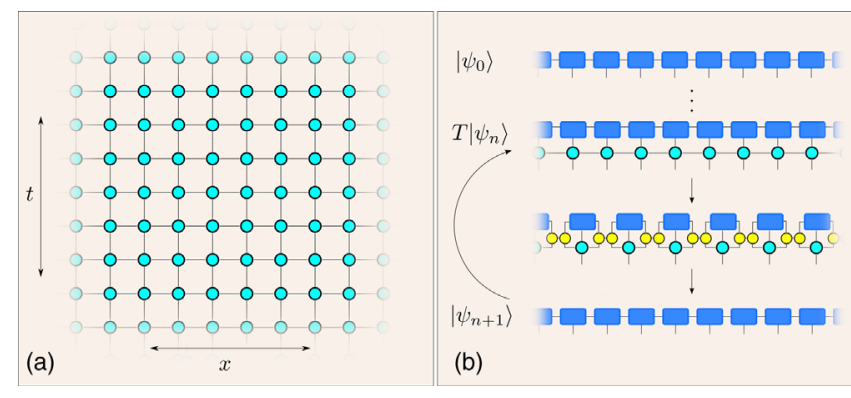

FIG. 2. (a) The ground space projector can be represented as an infinite 3D tensor network, spanning the three directions $x, y, t$. As usual, tensors are represented here by geometric shapes and lines attached to them are their indices. A line joining two tensors represents the tensor contraction. Here we represent a projection of the $3 \mathrm{D}$ tensor network in $2 \mathrm{D}$, where the vertical direction is the Euclidean time $t$, while the horizontal direction is the spatial direction $x$. (b) By using a boundary state we can approximate the full 3D network as the computation of the norm of a 2D iPEPS state. We contract one layer of the tensor network, the transfer matrix $T$, with the boundary iPEPS state $\left|\psi_{0}\right\rangle$ (first line). The bond dimension of the iPEPS state would increase exponentially number of time steps, and thus we approximate the iPEPS state by truncating its bond dimension back to $D$ (second to fourth line) using appropriate projectors (yellow tensors). We iterate these last two steps many times until the boundary state has actually converged. The converged iPEPS represents our best approximation of the ground state $|\Omega\rangle$ with finite bond dimension $D$. 
respect to the specific indices represented by the lines. The elementary tensors of the 3D network are obtained, for example, using the procedure described in the previous section and we assume they have horizontal bond dimension $d$. The transfer matrix $T$ here is represented by a single horizontal line of the network. One way to contract the network is to use boundary states $\left|\psi_{0}\right\rangle$ at both $t=-\infty$ and $t=+\infty$ that are already iPEPS. This strategy allows us to encode the full network contraction into a final iPEPS norm calculation. In Fig. 2, the boundary states are represented in blue. In the 2D cut the iPEPS boundary state looks like a $1 \mathrm{D}$ matrix product state with bond dimension $D$. We can now contract the network from $t=-\infty$ down to $t=0$ and simultaneously from $t=+\infty$ up to $t=0$. This is done iteratively by contracting at each time step the transfer matrix $T$ with the boundary iPEPS, corresponding to an evolution of the boundary iPEPS in imaginary time [7779]. At each step the bond dimension of the iPEPS increases by a factor $d$, and thus it increases exponentially with time. In order to keep the bond dimension of the boundary iPEPS finite, at each step we need to approximate the iPEPS states with another state with fixed bond dimension $D$. This is done by projecting the tensor product Hilbert space on the horizontal bonds having dimension $D d$ back to the original $D$. Practically, one needs to find optimal projectors that would perform such truncation (yellow tensors in Fig. 3). For the sake of our argument it is enough to assume that these projectors exist without providing the details on how to obtain them.

The procedure is repeated until the iPEPS state converges to a fixed point which encodes our best approximation of the ground state with an iPEPS with finite bond dimension $D$. By explicitly representing the action of the projectors on the original tensor network (as first suggested in Fig. 1 of Ref. [80]), we realize that the resulting tensor network looks very anisotropic. This is represented in Fig. 3. In both Figs. 3(a) and 3(b) the projectors are represented by yellow tensors. In Fig. 3(a) we show that by iteratively projecting the iPEPS states during the imaginary time evolution, we are actually creating almost one-dimensional channels along the time direction. If we now want to study the decay of correlations along the time direction, we need to characterize the decay of $\left\langle\Omega\left|\mathcal{O}\left(t_{0}\right) \mathcal{O}\left(t_{1}\right)\right| \Omega\right\rangle$. In Fig. 3(b) we represent the insertion of the two operators at certain times $t_{0}$ and $t_{1}$ by coloring in purple the corresponding tensors in the network. We immediately see that once the remaining part of the network is contracted into an effective environment (cyan rectangle), the computation is equivalent to a one-dimensional computation for the channel along the Euclidean time direction. Along this channel, the system is described by an effective matrix product state with finite bond dimension created by the projectors Fig. 3(b).

This immediately suggests that equal space, nonequal time correlation functions decay exponentially, since we already know that the $1 \mathrm{D}$ quantum system described by

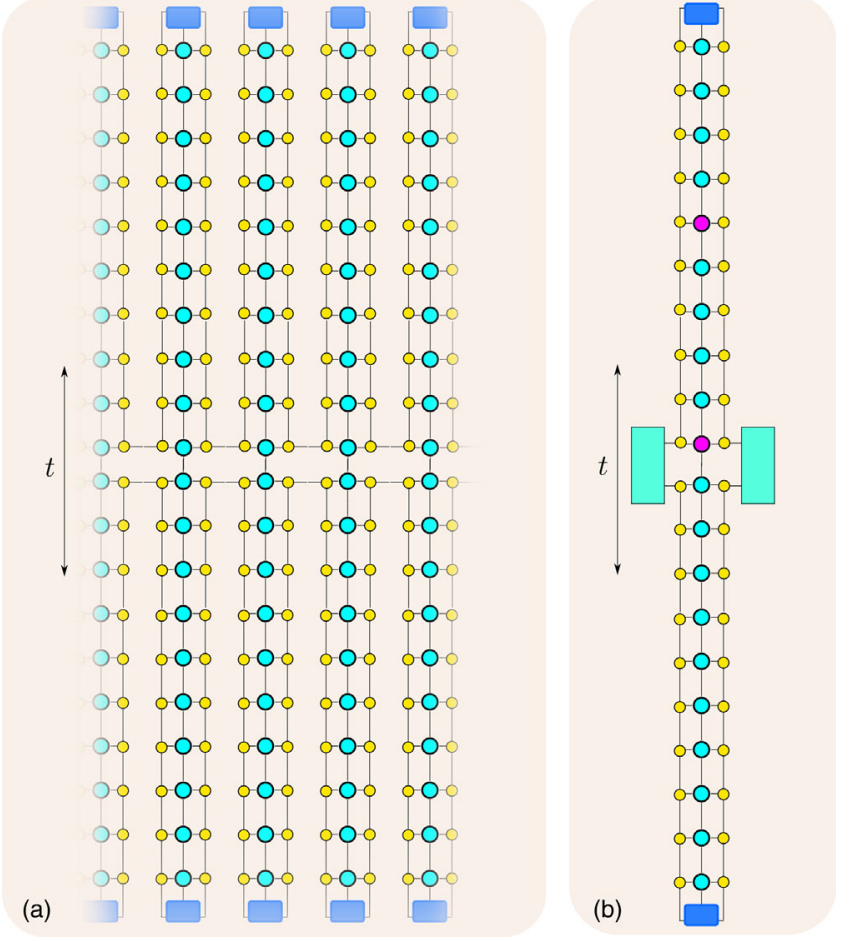

FIG. 3. (a) The fixed point iPEPS tensors are the result of many contraction and projection steps. Here we expand explicitly the iPEPS into its elementary constituents along the imaginary time direction (vertical in the figure), made by the original constituents of the 3D network (cyan tensors) and projectors (yellow tensors) used at each step to project the evolved iPEPS to a state with bond dimension $D$. The result is that the infinite 3D tensor network becomes very anisotropic, and we observe the appearance of quasi-one-dimensional time channels. (b) The equal space, nonequal time correlation function $\left\langle\Omega\left|\mathcal{O}\left(t_{0}\right) \mathcal{O}\left(t_{1}\right)\right| \Omega\right\rangle$. The insertion of the local operators $\mathcal{O}$ at two different times $t_{0}$ and $t_{1}$ is indicated by purple tensors. The contraction of the network outside the selected time channel produces the two environment tensors represented as rectangles on the left and the right of the time channel. The correlation function thus assumes a onedimensional structure. The projectors provide a timelike MPS state. Since the bond dimension of the MPS state is finite and equal to $D$, we expect that these correlations either do not decay or decay exponentially with the time separation $t_{1}-t_{0}$.

iMPS with finite bond dimension cannot be critical. The correlations in those states either do not decay or decay exponentially. This means that we expect $\left\langle\Omega\left|\mathcal{O}\left(t_{0}\right) \mathcal{O}\left(t_{1}\right)\right| \Omega\right\rangle \propto \exp \left[\left(-\left|t_{1}-t_{0}\right|\right) / \xi_{t}\right]$. Since these correlations are generated by powers of $T$ that is a function of $H$, the approximation scheme has introduced an effective finite correlation length in the imaginary time direction, as if the Hamiltonian was gapped. The approximation thus acts like a relevant perturbation to the critical Hamiltonian [81].

Thanks to the theorem proven by Hastings and Koma [82], the ground state of a gapped Hamiltonian has exponentially decaying correlation functions, and this we 
think is the physical origin of the finite correlation length we observe in our simulations.

This reasoning seems to suggest that the best iPEPS approximation to a critical ground state, in the scaling limit, where we can think of our system as a continuous system, transforms a smooth 3D solid geometry, into a "Swiss cheese," in which the size of holes (the valleys between different channels) is of the order of the lattice spacing and the correlation length along the channels actually provides the IR cutoff inducing all the phenomenology observed numerically. An illustration of this is sketched in Fig. 1.

A legitimate question is how to accommodate in this picture the existence of iPEPS with finite bond dimension and polynomially decaying correlations functions, such as those that are the ground state of generalized RK Hamiltonians. These states are known to describe a Lifshitz critical point, with low-energy dispersion relation $E(k) \propto k^{z}$ with $z \geq 2$ [47]. This implies that, contrary to what happens at Lorentz-invariant critical points, at Lifshitz points space and time play a very different role. In particular, these systems embed in 3D the 2D criticality of a classical system where the spatial correlations can be critical even if the imaginary time correlations are cut off by a finite inverse temperature. Whether a randomly initialized iPEPS would actually converge to a Lifshitz critical point, which is unstable under generic perturbation [47], is an interesting open question which we leave for future work.

\section{FINITE CORRELATION LENGTH SCALING}

Finite correlation length scaling (also known as finite entanglement scaling) has been introduced and applied in the context of infinite MPS [24-26] for the study of critical properties of $1 \mathrm{D}$ quantum systems, as an alternative to standard finite-size scaling using finite MPS. The basic idea is that the finite bond dimension $D$ induces a finite correlation length (a finite amount of entanglement), which can be used as a relevant length scale to perform a scaling analysis, in a very similar way as in the usual finite-size scaling approach, i.e., by replacing the system size $L$ by the effective correlation length at criticality $\xi_{D}$. For example, for an order parameter $m$, the ansatz reads

$$
m(g, L)=L^{-\beta / \nu} \mathcal{F}\left(g L^{1 / \nu}\right) \rightarrow \xi_{D}^{-\beta / \nu} \mathcal{M}\left(g \xi_{D}^{1 / \nu}\right)=m(g, D)
$$

where $g$ denotes the distance to the critical point and $\xi_{D}:=\xi(g=0, D)$.

A similar idea has also been used for 2D classical partition functions represented as a 2D tensor network [83], which are contracted using the CTM approach, where the finite boundary dimension $\chi$ introduces an effective correlation length $\xi_{\chi}$.
To what extent FCLS can also be applied to 2D quantum systems using iPEPS has not been explored yet. This generalization is also more challenging because the effective correlation length is affected by both the bond dimension $D$ of the ansatz and the boundary dimension $\chi$ used in the CTM contraction; i.e., in general scaling ansätze depend on both $\xi_{D}$ and $\xi_{\chi}$, e.g.,

$$
m(g, D, \chi)=\xi_{D}^{-\beta / \nu} \mathcal{M}\left(g \xi_{D}^{1 / \nu}, \xi_{D} / \xi_{\chi}\right)
$$

In order to solve this issue we eliminate the $\chi$ dependence by extrapolating the data in $\chi$, and perform a scaling analysis based on $\xi_{D}:=\xi(g=0, D, \chi \rightarrow \infty)$ only, so that the scaling ansatz reduces to Eq. (5). In the following we numerically demonstrate that this scaling ansatz can be used to extract critical properties in the $2 \mathrm{D}$ quantum case.

\section{SPINLESS FERMIONS ON THE HONEYCOMB LATTICE}

We consider a model of interacting spinless fermions on the honeycomb lattice at half filling, given by the Hamiltonian,

$$
\hat{H}=-t \sum_{\langle\mathbf{i}, \mathbf{j}\rangle}\left[\hat{c}_{\mathbf{i}}^{\dagger} \hat{c}_{\mathbf{j}}+\text { H.c. }\right]+V \sum_{\langle\mathbf{i}, \mathbf{j}\rangle} \hat{n}_{\mathbf{i}} \hat{n}_{\mathbf{j}}
$$

where the first term describes a nearest-neighbor hopping with amplitude $t$ and the second term a repulsive nearestneighbor interaction with strength $V$, with $\hat{n}_{i}=\hat{c}_{\mathrm{i}}^{\dagger} \hat{c}_{\mathbf{i}}$. This model has been intensely studied in the past [84-89] and is known to undergo a continuous phase transition between a Dirac semimetal phase and a charge-density wave (CDW) phase at a critical coupling of $V_{c} / t=1.356$ (1) [84]. In the continuum limit the transition belongs to the chiral Ising Gross-Neveu universality class with a dynamical critical exponent $z=1$.

The CDW order parameter is given by

$$
m=\left|n_{A}-n_{B}\right|,
$$

where $n_{A}$ and $n_{B}$ are the particle densities on sublattices $A$ and $B$, respectively. Figure 4(a) shows the order parameter as a function of interaction strength $V / t$ for different bond dimensions $D$, where one can clearly observe the finite- $D$ effects, i.e., no sharp phase transition at $V_{c} / t=1.356$, but a systematic suppression of $m$ with increasing $D$, very similar to standard finite-size effects.

We first test the scaling ansatz at the critical point, i.e., $g=\left(V-V_{c}\right) / V_{c}=0$, with $V_{c} / t=1.356$, where Eq. (2) reduces to

$$
m(g=0, D, \chi)=\xi_{D}^{-\beta / \nu} \mathcal{M}\left(0 \cdot \xi_{D}^{1 / \nu}, \xi_{\chi} / \xi_{D}\right) \sim \xi_{D}^{-\beta / \nu}
$$



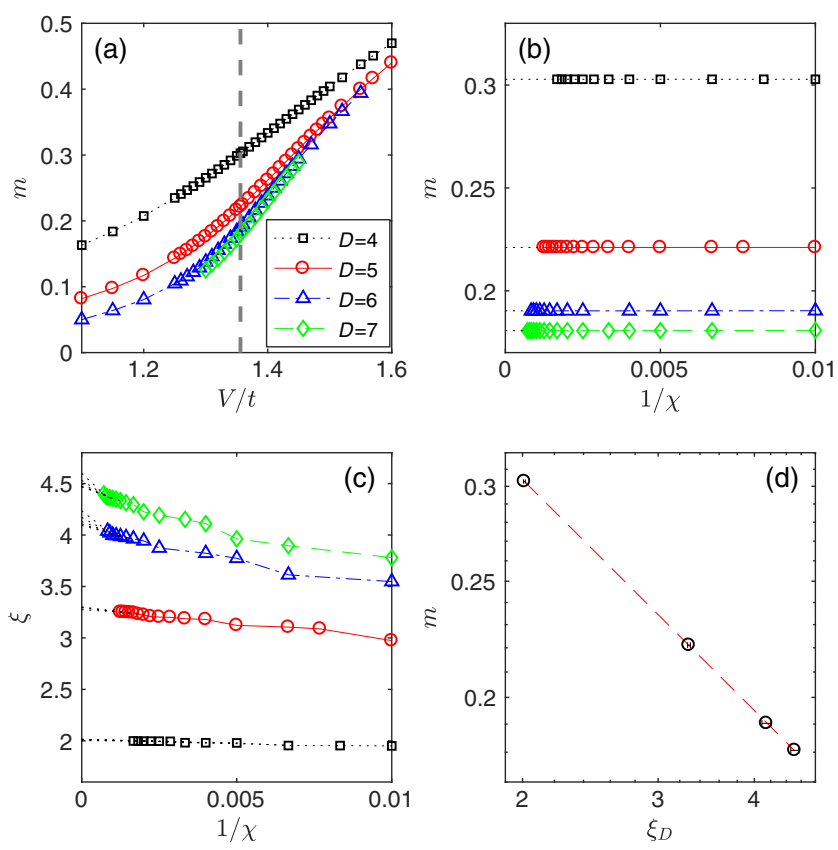

FIG. 4. (a) CDW order parameter as a function of interaction strength $V / t$ for different values of $D$. The location of the critical point is indicated by the dashed line. (b) Order parameter $m$ and (c) correlation length $\xi$ as a function of inverse boundary dimension $\chi$ for different values of $D$ at the critical point $V / t=1.356$. (d) A power-law fit at the critical point yields an exponent $\beta / \nu=0.64(2)$, in agreement with the $\mathrm{QMC}$ result.

The latter relation is obtained by taking a sufficiently large $\chi$ such that $m$ is fully converged in $\chi$. Figure 4(b) shows that $m$ converges rapidly in $\chi$ such that no extrapolation in $\chi$ is needed. However, the correlation length $\xi$ displays a stronger dependence on $\chi$ [90], shown in Fig. 4(c). We determine $\xi_{D}$ for each value of $D$ by extrapolating $\xi(D, \chi)$ to the infinite $\chi$ limit. We do this by performing linear extrapolations in $1 / \chi$ using different ranges of data points, and determining the average and standard deviation of these extrapolations [91]. We clearly find that even at the largest bond dimension the state is not critical, i.e., that the finite $D$ induces a finite correlation length, similarly as in 1D with MPS. Finally, Fig. 4(d) shows a log-log plot of $m$ versus $\xi_{D}$, where a linear fit yields an estimate for $\beta / \nu=$ $0.64(2)$ in agreement with the $\mathrm{QMC}$ result $\beta / \nu=0.65(4)$ from Ref. [84].

Next, we check if we can consistently determine the critical point $V_{c}$ based on this result for $\beta / \nu$. Using the scaling ansatz Eq. (2) at the critical point in the large $\chi$ limit, we obtain

$$
y=m(g=0, D, \chi \rightarrow \infty) \xi_{D}^{\beta / \nu}=\mathrm{const},
$$

i.e., a constant independent of $D$. In Fig. 5(a), we plot $y$ as a function of $D$ for different values of $V / t$, and Fig. 5(b) shows the deviation from a straight line (computed by the
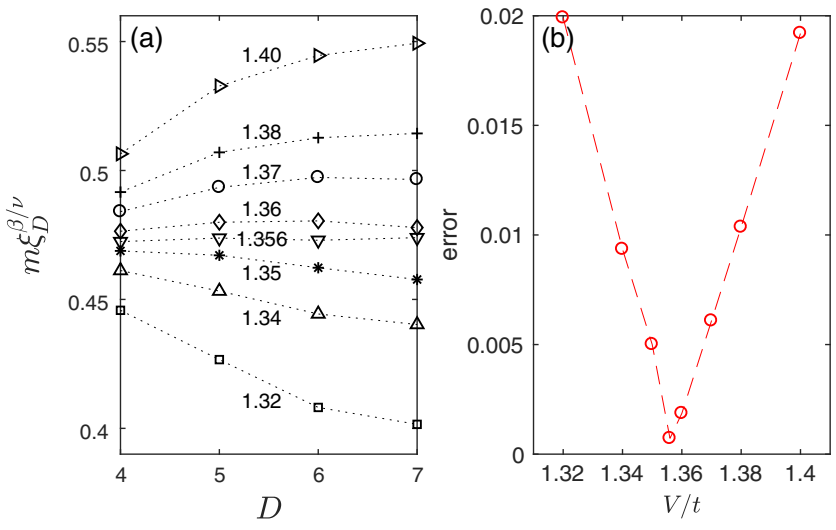

FIG. 5. Results for $y=m \xi^{\beta / \nu}$ as a function of $D$ for fixed $\beta / \nu=0.64$. At the critical point $V_{c} / t=1.356$ the value of $y$ becomes independent of the bond dimension $D$, cf. Eq. (6). The standard deviation of the points is shown on the right-hand side, which clearly exhibits a minimum at the critical point.

standard deviation of $y$ ) as a function of $V / t$ (where $\xi_{D}$ is determined for each value of $V / t$ ). The smallest deviation is obtained for $V / t=1.356$, consistent with the QMC result. (A similar result is also obtained using $\beta / \nu=0.65$.)

We next attempt to perform a data collapse using the following two ansätze (again in the large $\chi$ limit),

$$
\begin{aligned}
& m(g, D) \xi_{D}^{\beta / \nu}=\mathcal{M}\left(g \xi_{D}^{1 / \nu}\right), \\
& m(g, D) g^{-\beta}=\tilde{\mathcal{M}}\left(g \xi_{D}^{1 / \nu}\right),
\end{aligned}
$$

to determine the critical exponents $\nu$ and $\beta$ (with $\left.V_{c} / t=1.356\right)$, shown in Fig. 6. By varying the range of data points and by taking the error bar of $\xi_{D}$ into account, we obtain the following estimates: $\beta=0.51(1)$ and $\nu=$ $0.79(2)$, in agreement with the QMC result $\beta=0.52(3)$, $\nu=0.80(3)[84]$.

\section{A. Determining the critical point based on $\boldsymbol{m}^{\prime} / \boldsymbol{m}$}

A standard method to determine the critical point without knowledge of the critical exponents is by the Binder cumulant, which is invariant at the critical point for different system sizes (or bond dimensions). However, this
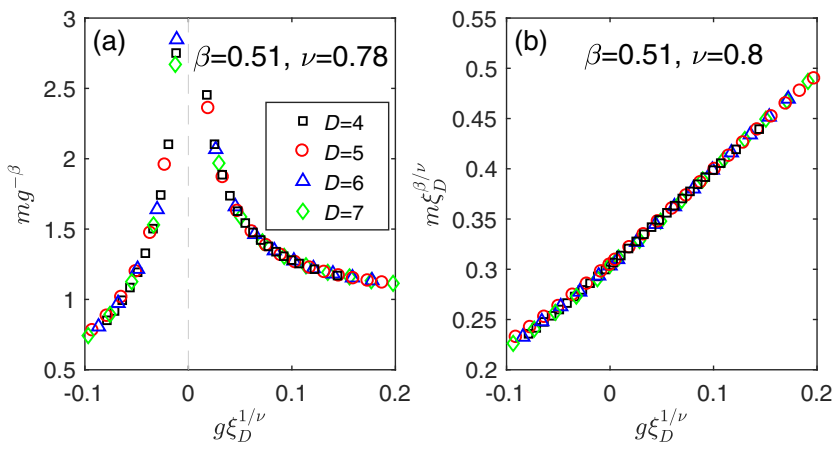

FIG. 6. Data collapse plots using Eqs. (7) and (8). 
would require computing the fourth-order moment of the order parameter, which is difficult in a $2 \mathrm{D}$ tensor network approach. Here we introduce an alternative approach based on the derivative of the order parameter with respect to $g$, which in the large $\chi$ limit is expected to obey the following ansatz:

$$
m^{\prime}(g, D)=\xi_{D}^{-(\beta-1) / \nu} \mathcal{M}^{\prime}\left(g \xi_{D}^{1 / \nu}\right)
$$

Thus, at the critical point, $g=0$,

$$
\frac{m_{c}^{\prime}(D)}{m_{c}(D)}:=\frac{m^{\prime}(g=0, D)}{m(g=0, D)} \sim \xi_{D}^{1 / \nu}
$$

Thus, we have found an expression for $\xi_{D}^{1 / \nu}$ which we can use to rewrite scaling functions:

$$
\mathcal{M}\left(g \xi_{D}^{1 / \nu}\right) \sim \mathcal{P}\left(g \frac{m_{c}^{\prime}(D)}{m_{c}(D)}\right) .
$$

Dividing Eq. (9) by $m$ and multiplying by $g$ yields

$$
g \frac{m^{\prime}(g, D)}{m(g, D)}=\overline{\mathcal{M}}^{\prime}\left(g \xi_{D}^{1 / \nu}\right)=\mathcal{P}\left(g \frac{m_{c}^{\prime}(D)}{m_{c}(D)}\right) .
$$

Thus, we can plot $y=\left(V-V_{c}\right)\left\{\left[\left(m^{\prime}(g, D)\right] /[m(g, D)]\right\}\right.$ versus $x=\left(V-V_{c}\right)\left\{\left[m_{c}^{\prime}(D)\right] /\left[m_{c}(D)\right]\right\}$ as a function of $V$, and for the correct choice of $V_{c}$ the data for different values of $D$ collapse onto a single curve. The best data collapse, shown in Fig. 7(a), is obtained for $V_{c} / t=$ $1.356(2)$ in agreement with the QMC result. The numerical derivative has been obtained by taking the derivative of polynomial fits to $m$ versus $V / t$ data.

Finally, we can combine Eqs. (11) and (8),

$$
m(g, D) g^{-\beta}=\tilde{\mathcal{P}}\left(g \frac{m_{c}^{\prime}(D)}{m_{c}(D)}\right)
$$

to perform a data collapse and extract the exponent $\beta$, shown in Fig. 7, yielding a consistent value of $\beta=0.53(1)$.
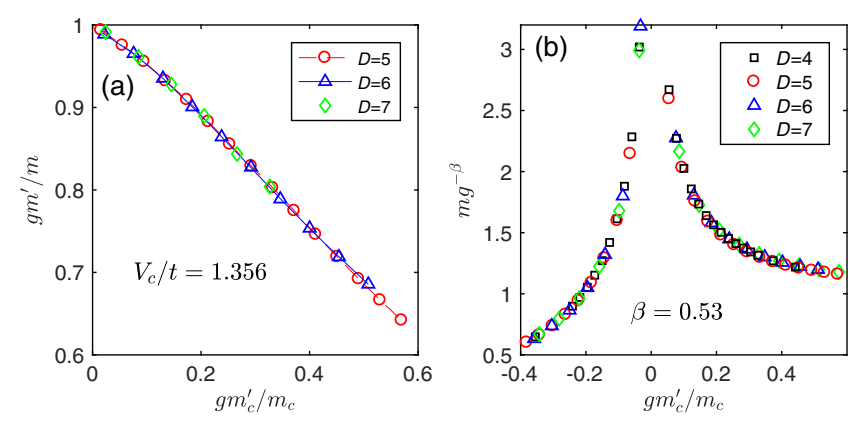

FIG. 7. (a) Data collapse using Eq. (12) yielding $V_{c} / t=1.356(2)$. (b) Data collapse based on Eq. (13) giving $\beta=0.53(1)$.
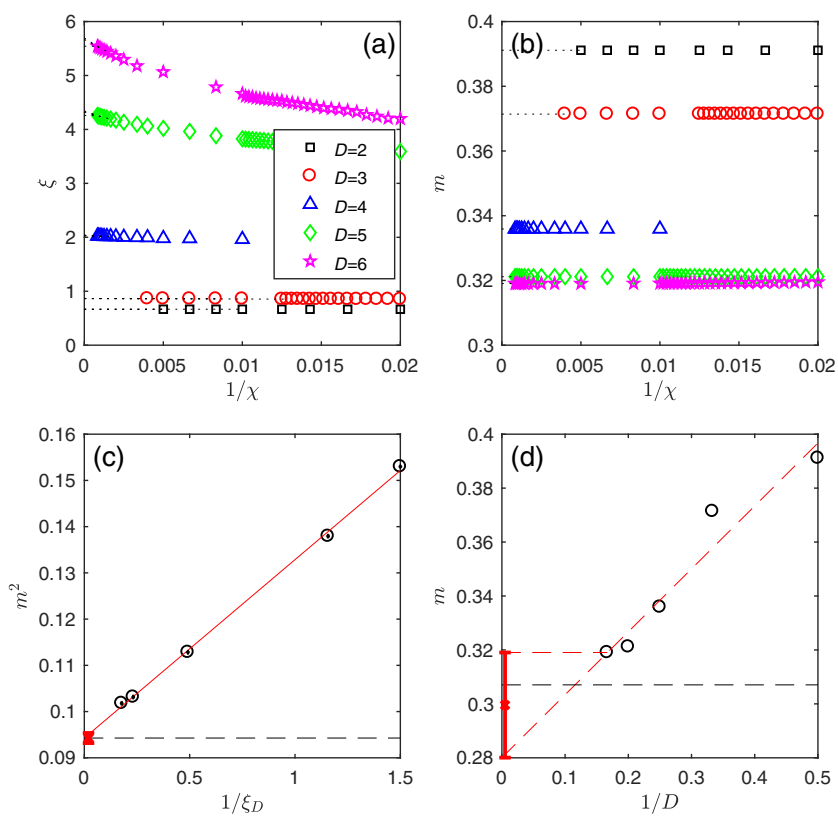

FIG. 8. iPEPS results for the 2D Heisenberg model. (a) Correlation length $\xi$ and (b) staggered magnetization $m$ as a function of inverse $\chi$ for different values of $D$. (c) Squared of the staggered magnetization as a function of inverse $\xi_{D}$. A linear extrapolation yields $m=0.307 \pm 0.002$, which is in agreement with the QMC result $m=0.30743(1)$. (d) Order parameter as a function of inverse $D$.

\section{IMPROVED EXTRAPOLATIONS OF ORDER PARAMETERS}

The effective correlation length $\xi_{D}$ can also be used to perform an improved extrapolation of the order parameter in a gapless system. As an illustration, we present results for the two-dimensional $S=1 / 2$ Heisenberg model on a square lattice. Figures 8(a) and 8(b) show the correlation length $\xi$ and staggered magnetization $m$ as a function of inverse $\chi$, respectively. A linear extrapolation of $m^{2}$ as a function of $\xi_{D}$ in Fig. 8(c) yields $m=0.307 \pm 0.002$, in agreement with the state-of-the-art QMC result $m=$ 0.30743 (1) from Ref. [93]. This approach provides a much better estimate than the one based on a crude $1 / D$ extrapolation shown in Fig. 8(d), which is not very accurate due to the nonregular behavior as a function of $D$.

\section{SUMMARY AND CONCLUSION}

In this paper, we have demonstrated the usefulness and applicability of finite correlation length scaling in two dimensions based on iPEPS by determining the critical exponents and critical point of an interacting spinless fermion model on a honeycomb lattice. Our findings are in agreement with the QMC results from Ref. [84]. Furthermore, we introduced a new approach to determine the critical point based on the derivative of the order parameter, which does not require the computation of higher-order moments of the order 
parameter or extrapolations of the effective correlation length in $\chi$, making it a particularly useful approach for $2 \mathrm{D}$ tensor network calculations.

We stress that iPEPS can also be applied to models which are inaccessible to QMC simulations due to the negative sign problem, making it a promising tool to study critical properties of challenging open problems.

From the theoretical point of view, we have possibly identified a class of models whose ground states are hard to encode with iPEPS with finite bond dimension, despite obeying the area law of entanglement. We have given an intuitive argument that the mismatch between the exact critical states and the finitely correlated iPEPS we obtain has a geometric origin. The iPEPS seems to approximate the smooth continuous 3D geometry with a landscape of towers separated by valleys, where the finite correlation time along the towers could be the ultimate reason for the appearance of the finite correlation length.

Our results are also relevant to field theories. The operator content of a field theory is known to depend on the geometry and the boundary conditions of the space on which the theory is defined [94,95]. It would be important to understand the effects of the landscape of towers and valleys on the operator content of the corresponding field theory. Furthermore, our results seem to suggest that $D$ would act as a regulator in any continuum limit of the theory defined on the lattice. The bond dimension of the iPEPS should indeed increase in order to keep the ratio between the relevant physical quantities and the correlation length fixed as we approach the continuum limit.

\section{ACKNOWLEDGMENTS}

We acknowledge discussions with Andreas Läuchli and Michael Rader. L. T. acknowledges the long-time discussions and collaboration with Andrea Coser on similar problems studied in the context of Gaussian tensor networks. This project was supported by the European Research Council (ERC) under the European Unions Horizon 2020 research and innovation programme (Grant Agreement No. 677061), and by the Narodowe Centrum Nauki (National Science Center) under Project No. 2016/23/B/ ST3/00830. Work at the University of Strathclyde was supported by the EPSRC Programme GrantDesOEQ (EP/ P009565/1). This project is part of the Delta-ITP consortium, a program of the Netherlands Organization for Scientific Research (NWO) that is funded by the Dutch Ministry of Education, Culture and Science (OCW).

Note added.-Similar results have been reported by Rader and Läuchli [96].

[1] F. Verstraete, V. Murg, and J. I. Cirac, Matrix Product States, Projected Entangled Pair States, and Variational
Renormalization Group Methods for Quantum Spin Systems, Adv. Phys. 57, 143 (2008).

[2] U. Schollwöck, The Density-Matrix Renormalization Group in the Age of Matrix Product States, Ann. Phys. (Amsterdam) 326, 96 (2011).

[3] R. Orús, A Practical Introduction to Tensor Networks: Matrix Product States and Projected Entangled Pair States, Ann. Phys. (Amsterdam) 349, 117 (2014).

[4] J. C. Bridgeman and C. T. Chubb, Hand-Waving and Interpretive Dance: An Introductory Course on Tensor Networks, J. Phys. A 50, 223001 (2017).

[5] J. Haegeman and F. Verstraete, Diagonalizing Transfer Matrices and Matrix Product Operators: A Medley of Exact and Computational Methods, Annu. Rev. Condens. Matter Phys. 8, 355 (2017).

[6] M. B. Hastings, An Area Law for One-Dimensional Quantum Systems, J. Stat. Mech. (2007) P08024.

[7] M. M. Wolf, F. Verstraete, M. B. Hastings, and J. I. Cirac, Area Laws in Quantum Systems: Mutual Information and Correlations, Phys. Rev. Lett. 100, 070502 (2008).

[8] J. Eisert, M. Cramer, and M. B. Plenio, Colloquium: Area Laws for the Entanglement Entropy, Rev. Mod. Phys. 82, 277 (2010).

[9] L. Amico, R. Fazio, A. Osterloh, and V. Vedral, Entanglement in Many-Body Systems, Rev. Mod. Phys. 80, 517 (2008).

[10] L. Masanes, Area Law for the Entropy of Low-Energy States, Phys. Rev. A 80, 052104 (2009).

[11] N. Laflorencie, Quantum Entanglement in Condensed Matter Systems, Phys. Rep. 646, 1 (2016).

[12] I. Affleck, T. Kennedy, E. H. Lieb, and H. Tasaki, Rigorous Results on Valence-Bond Ground States in Antiferromagnets, Phys. Rev. Lett. 59, 799 (1987).

[13] S. Ostlund and S. Rommer, Thermodynamic Limit of Density Matrix Renormalization, Phys. Rev. Lett. 75, 3537 (1995).

[14] D. Perez-Garcia, F. Verstraete, M. M. Wolf, and J. I. Cirac, Matrix Product State Representations, Quantum Inf. Comput. 7, 401 (2007).

[15] F. Verstraete and J. I Cirac, Renormalization Algorithms for Quantum-Many Body Systems in Two and Higher Dimensions, arXiv:cond-mat/0407066.

[16] J. Jordan, R. Orús, G. Vidal, F. Verstraete, and J. I. Cirac, Classical Simulation of Infinite-Size Quantum Lattice Systems in Two Spatial Dimensions, Phys. Rev. Lett. 101, 250602 (2008).

[17] T. Nishino, Y. Hieida, K. Okunishi, N. Maeshima, Y. Akutsu, and A. Gendiar, Two-Dimensional Tensor Product Variational Formulation, Prog. Theor. Phys. 105, 409 (2001).

[18] Y. Nishio, N. Maeshima, A. Gendiar, and T. Nishino, Tensor Product Variational Formulation for Quantum Systems, arXiv:cond-mat/0401115.

[19] C. G. Callan and F. Wilczek, On Geometric Rntropy, Phys. Lett. B 333, 55 (1994).

[20] T. J. Osborne and M. A. Nielsen, Entanglement in a Simple Quantum Phase Transition, Phys. Rev. A 66, 032110 (2002).

[21] P. Calabrese and J. Cardy, Entanglement Entropy and Quantum Field Theory, J. Stat. Mech. (2004) P06002. 
[22] P. Calabrese and J. Cardy, Entanglement Entropy and Conformal Field Theory, J. Phys. A 42, 504005 (2009).

[23] G. Vidal, J. I. Latorre, E. Rico, and A. Kitaev, Entanglement in Quantum Critical Phenomena, Phys. Rev. Lett. 90, 227902 (2003).

[24] L. Tagliacozzo, T. R. de Oliveira, S. Iblisdir, and J. I. Latorre, Scaling of Entanglement Support for Matrix Product States, Phys. Rev. B 78, 024410 (2008).

[25] F. Pollmann, S. Mukerjee, A. M. Turner, and J. E. Moore, Theory of Finite-Entanglement Scaling at One-Dimensional Quantum Critical Points, Phys. Rev. Lett. 102, 255701 (2009).

[26] B. Pirvu, G. Vidal, F. Verstraete, and L. Tagliacozzo, Matrix Product States for Critical Spin Chains: Finite-Size versus Finite-Entanglement Scaling, Phys. Rev. B 86, 075117 (2012).

[27] H. Ueda, K. Okunishi, and T. Nishino, Doubling of Entanglement Spectrum in Tensor Renormalization Group, Phys. Rev. B 89, 075116 (2014).

[28] There have been promising developments in analytical bootstrap [29,30], but the accuracy has not yet reached the level of numerical calculations (see, e.g., Refs. [31-34]).

[29] S. Rychkov and Z.M. Tan, The e-Expansion from Conformal Field Theory, J. Phys. A 48, 29FT01 (2015).

[30] F. Gliozzi, A. Guerrieri, A. C. Petkou, and C. Wen, Generalized Wilson-Fisher Critical Points from the Conformal OPE, Phys. Rev. Lett. 118, 061601 (2017).

[31] D. Simmons-Duffin, A Semidefinite Program Solver for the Conformal Bootstrap, J. High Energy Phys. 06 (2015) 174.

[32] F. Kos, D. Poland, D. Simmons-Duffin, and A. Vichi, Precision Islands in the Ising and $O(N)$ Models, J. High Energy Phys. 08 (2016) 36.

[33] F. Gliozzi, Constraints on Conformal Field Theories in Diverse Dimensions from the Bootstrap Mechanism, Phys. Rev. Lett. 111, 161602 (2013).

[34] F. Gliozzi, P. Liendo, M. Meineri, and A. Rago, Boundary and Interface CFTs from the Conformal Bootstrap, J. High Energy Phys. 05 (2015) 36.

[35] W. Li, L. Ding, R. Yu, T. Roscilde, and S. Haas, Scaling Behavior of Entanglement in Two- and Three-Dimensional Free-Fermion Systems, Phys. Rev. B 74, 073103 (2006).

[36] H. Casini and M. Huerta, Universal Terms for the Entanglement Entropy in 2+1 Dimensions, Nucl. Phys. B764, 183 (2007).

[37] T. Nishioka, S. Ryu, and T. Takayanagi, Holographic Entanglement Entropy: An Overview, J. Phys. A 42, 504008 (2009).

[38] L. Tagliacozzo, G. Evenbly, and G. Vidal, Simulation of Two-Dimensional Quantum Systems Using a Tree Tensor Network that Exploits the Entropic Area Law, Phys. Rev. B 80, 235127 (2009).

[39] M. A. Metlitski, C. A. Fuertes, and S. Sachdev, Entanglement Entropy in the $O(N)$ Model, Phys. Rev. B 80, 115122 (2009).

[40] C. V. Kraus, N. Schuch, F. Verstraete, and J. I. Cirac, Fermionic Projected Entangled Pair States, Phys. Rev. A 81, 052338 (2010).

[41] D. Poilblanc and M. Mambrini, Quantum Critical Phase with Infinite Projected Entangled Paired States, Phys. Rev. B 96, 014414 (2017).
[42] F. Verstraete, M. M. Wolf, D. Perez-Garcia, and J. I. Cirac, Criticality, the Area Law, and the Computational Power of Projected Entangled Pair States, Phys. Rev. Lett. 96, 220601 (2006).

[43] For example, the 2D classical partition function of the critical 2D Ising model represented as an iPEPS with $D=2$.

[44] C. L. Henley, From Classical to Quantum Dynamics at Rokhsar-Kivelson Points, J. Phys. Condens. Matter 16, S891 (2004).

[45] E. Ardonne, P. Fendley, and E. Fradkin, Topological Order and Conformal Quantum Critical Points, Ann. Phys. (Amsterdam) 310, 493 (2004).

[46] C. Castelnovo, C. Chamon, C. Mudry, and P. Pujol, From Quantum Mechanics to Classical Statistical Physics: Generalized Rokhsar-Kivelson Hamiltonians and the "Stochastic Matrix Form" Decomposition, Ann. Phys. (Amsterdam) 318, 316 (2005).

[47] S. V. Isakov, P. Fendley, A. W. W. Ludwig, S. Trebst, and M. Troyer, Dynamics at and Near Conformal Quantum Critical Points, Phys. Rev. B 83, 125114 (2011).

[48] L. Tagliacozzo, A. Celi, and M. Lewenstein, Tensor Networks for Lattice Gauge Theories with Continuous Groups, Phys. Rev. X 4, 041024 (2014).

[49] E. Zohar, M. Burrello, T. B. Wahl, and J. I. Cirac, Fermionic Projected Entangled Pair States and Local U(1) Gauge Theories, Ann. Phys. (Amsterdam) 363, 385 (2015).

[50] E. Zohar, T. B. Wahl, M. Burrello, and J. I. Cirac, Projected Entangled Pair States with Non-Abelian Gauge Symmetries: An SU(2) Study, Ann. Phys. (Amsterdam) 374, 84 (2016).

[51] S.-J. Ran, C. Peng, W. Li, M. Lewenstein, and G. Su, Criticality in Two-Dimensional Quantum Systems: Tensor Network Approach, Phys. Rev. B 95, 155114 (2017).

[52] H. J. Liao, Z. Y. Xie, J. Chen, X. J. Han, H. D. Xie, B. Normand, and T. Xiang, Heisenberg Antiferromagnet on the Husimi Lattice, Phys. Rev. B 93, 075154 (2016).

[53] H. J. Liao, Z. Y. Xie, J. Chen, Z. Y. Liu, H. D. Xie, R. Z. Huang, B. Normand, and T. Xiang, Gapless Spin-Liquid Ground State in the $S=1 / 2$ Kagome Antiferromagnet, Phys. Rev. Lett. 118, 137202 (2017).

[54] Y. Ge and J. Eisert, Area Laws and Efficient Descriptions of Quantum Many-Body States, New J. Phys. 18, 083026 (2016).

[55] H. H. Zhao, C. Xu, Q. N. Chen, Z. C. Wei, M. P. Qin, G. M. Zhang, and T. Xiang, Plaquette Order and Deconfined Quantum Critical Point in the Spin-1 Bilinear-Biquadratic Heisenberg Model on the Honeycomb Lattice, Phys. Rev. B 85, 134416 (2012).

[56] P. Corboz, M. Lajkó, A. M. Läuchli, K. Penc, and F. Mila, Spin-Orbital Quantum Liquid on the Honeycomb Lattice, Phys. Rev. X 2, 041013 (2012).

[57] Z. Y. Xie, J. Chen, J. F. Yu, X. Kong, B. Normand, and T. Xiang, Tensor Renormalization of Quantum Many-Body Systems Using Projected Entangled Simplex States, Phys. Rev. X 4, 011025 (2014).

[58] P. Corboz and F. Mila, Tensor Network Study of the ShastrySutherland Model in Zero Magnetic Field, Phys. Rev. B 87, 115144 (2013).

[59] Z.-C. Gu, H.-C. Jiang, D. N. Sheng, H. Yao, L. Balents, and X.-G. Wen, Time-Reversal Symmetry Breaking 
Superconducting Ground State in the Doped Mott Insulator on the Honeycomb Lattice, Phys. Rev. B 88, 155112 (2013).

[60] P. Corboz, T. M. Rice, and Matthias Troyer, Competing States in the t-J Model: Uniform d-Wave State versus Stripe State, Phys. Rev. Lett. 113, 046402 (2014).

[61] T. Picot and D. Poilblanc, Nematic and Supernematic Phases in Kagome Quantum Antiferromagnets under the Influence of a Magnetic Field, Phys. Rev. B 91, 064415 (2015).

[62] T. Picot, M. Ziegler, R. Orús, and D. Poilblanc, Spin-S Kagome Quantum Antiferromagnets in a Field with Tensor Networks, Phys. Rev. B 93, 060407 (2016).

[63] H. J. Liao, Z. Y. Xie, J. Chen, Z. Y. Liu, H. D. Xie, R. Z. Huang, B. Normand, and T. Xiang, Gapless Spin-Liquid Ground State in the $S=1 / 2$ Kagome Antiferromagnet, Phys. Rev. Lett. 118, 137202 (2017).

[64] I. Niesen and P. Corboz, Emergent Haldane Phase in the $S=1$ Bilinear-Biquadratic Heisenberg Model on the Square Lattice, Phys. Rev. B 95, 180404 (2017).

[65] B.-X. Zheng, C.-M. Chung, P. Corboz, G. Ehlers, M.-P. Qin, R. M. Noack, H. Shi, S. R. White, S. Zhang, and G. K.-L. Chan, Stripe Order in the Underdoped Region of the TwoDimensional Hubbard Model, Science 358, 1155 (2017).

[66] R. Haghshenas and D. N. Sheng, The U(1)-Symmetric Infinite Projected Entangled-Pair State Study of the Spin-1/2 Square $J_{1}-J_{2}$ Heisenberg Model, Phys. Rev. B 97, 174408 (2018).

[67] P. Corboz, R. Orus, B. Bauer, and G. Vidal, Simulation of Strongly Correlated Fermions in Two Spatial Dimensions with Fermionic Projected Entangled-Pair States, Phys. Rev. B 81, 165104 (2010).

[68] M. Lubasch, J. I. Cirac, and M.-C. Bañuls, Algorithms for Finite Projected Entangled Pair States, Phys. Rev. B 90, 064425 (2014).

[69] H. N. Phien, J. A. Bengua, H. D. Tuan, P. Corboz, and R. Orus, Infinite Projected Entangled Pair States Algorithm Improved: Fast Full Update and Gauge Fixing, Phys. Rev. B 92, 035142 (2015).

[70] P. Corboz, Variational Optimization with Infinite Projected Entangled-Pair States, Phys. Rev. B 94, 035133 (2016).

[71] L. Vanderstraeten, J. Haegeman, P. Corboz, and F. Verstraete, Gradient Methods for Variational Optimization of Projected Entangled-Pair States, Phys. Rev. B 94, 155123 (2016).

[72] T. Nishino and K. Okunishi, Corner Transfer Matrix Renormalization Group Method, J. Phys. Soc. Jpn. 65, 891 (1996).

[73] R. Orús and G. Vidal, Simulation of Two-Dimensional Quantum Systems on an Infinite Lattice Revisited: Corner Transfer Matrix for Tensor Contraction, Phys. Rev. B 80, 094403 (2009).

[74] S. Singh, R. N. C. Pfeifer, and G. Vidal, Tensor Network States and Algorithms in the Presence of a Global U(1) Symmetry, Phys. Rev. B 83, 115125 (2011).

[75] B. Bauer, P. Corboz, R. Orús, and M. Troyer, Implementing Global Abelian Symmetries in Projected Entangled-Pair State Algorithms, Phys. Rev. B 83, 125106 (2011).

[76] If $H$ is gapless, a projector onto a well-defined state can be obtained by first opening an infinitesimally small gap of order $\epsilon$ modifying $H \rightarrow H_{\epsilon}$, and then taking the limit $\beta \rightarrow \infty$. We avoid similar details in the rest of the section.
[77] G. Vidal, Classical Simulation of Infinite-Size Quantum Lattice Systems in One Spatial Dimension, Phys. Rev. Lett. 98, 070201 (2007).

[78] G. Vidal, Efficient Simulation of One-Dimensional Quantum Many-Body Systems, Phys. Rev. Lett. 93, 040502 (2004).

[79] R. Orús and G. Vidal, Infinite Time-Evolving Block Decimation Algorithm beyond Unitary Evolution, Phys. Rev. B 78, 155117 (2008).

[80] A. J. Ferris, Unbiased Monte Carlo for the Age of Tensor Networks, arXiv:1507.00767.

[81] J. Cardy, Scaling and Renormalization in Statistical Physics (Cambridge University Press, Cambridge, England, 1996).

[82] M. B. Hastings and T. Koma, Spectral Gap and Exponential Decay of Correlations, Commun. Math. Phys. 265, 781 (2006).

[83] T. Nishino, K. Okunishi, and M. Kikuchi, Numerical Renormalization Group at Criticality, Phys. Lett. A 213, 69 (1996).

[84] L. Wang, P. Corboz, and M. Troyer, Fermionic Quantum Critical Point of Spinless Fermions on a Honeycomb Lattice, New J. Phys. 16, 103008 (2014).

[85] D. D. Scherer, M. M. Scherer, and C. Honerkamp, Correlated Spinless Fermions on the Honeycomb Lattice Revisited, Phys. Rev. B 92, 155137 (2015).

[86] Z.-X. Li, Y.-F. Jiang, and H. Yao, Fermion-Sign-Free Majarana-Quantum-Monte-Carlo Studies of Quantum Critical Phenomena of Dirac Fermions in Two Dimensions, New J. Phys. 17, 085003 (2015).

[87] L. Wang, M. Iazzi, P. Corboz, and M. Troyer, Efficient Continuous-Time Quantum Monte Carlo Method for the Ground State of Correlated Fermions, Phys. Rev. B 91, 235151 (2015).

[88] L. Wang, Y.-H. Liu, and M. Troyer, Stochastic Series Expansion Simulation of the $t-V$ Model, Phys. Rev. B 93, 155117 (2016).

[89] S. Capponi, Phase Diagram of Interacting Spinless Fermions on the Honeycomb Lattice, J. Phys. Condens. Matter 29, 043002 (2017).

[90] The correlation length is computed from the largest and second largest eigenvalues of the row-to-row transfer matrix as in Ref. [83]. In the honeycomb model the correlation length is measured in units of the $2 \times 2$ unit cell size.

[91] Recently, an interesting alternative approach to obtain the correlation length in the infinite $\chi$ limit was introduced in Ref. [92].

[92] M. M. Rams, P. Czarnik, and L. Cincio, Precise Extrapolation of the Correlation Function Asymptotics in Uniform Tensor Network States with Application to the BoseHubbard and XXZ Models, arXiv:1801.08554.

[93] A. W. Sandvik and H. G. Evertz, Loop Updates for Variational and Projector Quantum Monte Carlo Simulations in the Valence-Bond Basis, Phys. Rev. B 82, 024407 (2010).

[94] J. L. Cardy, Operator Content of Two-Dimensional Conformally Invariant Theories, Nucl. Phys. B270, 186 (1986).

[95] J. L. Cardy, Effect of Boundary Conditions on the Operator Content of Two-Dimensional Conformally Invariant Theories, Nucl. Phys. B275, 200 (1986).

[96] M. Rader and A. Läuchli, preceding article, Finite Correlation Length Scaling in Lorentz-Invariant Gapless iPEPS Wave Functions, Phys. Rev. X 8, 031030 (2018). 